\title{
Optimal Admission and Eviction Control of Secondary Users at Cognitive Radio HotSpots
}

\author{
Hyoil Kim and Kang G. Shin \\ Real-Time Computing Laboratory \\ EECS Department, The University of Michigan \\ Ann Arbor, Michigan 48109-2121 \\ Email: \{hyoilkim,kgshin\}@eecs.umich.edu
}

\begin{abstract}
Cognitive Radio (CR) HotSpot is a typical example of commercialized Dynamic Spectrum Access (DSA) in which a Wireless Service Provider (WSP) provides CR end-users access to its local-area network. A WSP temporarily leases licensed channels from the primary licensees via spectrum auction, and subleases them to CR end-users or customers, each demanding a different amount of spectrum, according to its own advertised pricing policy. The CR customers use the subleased channels opportunistically when the channels are not being used by primary/legacy users, and hence, the amount of available spectrum resource varies with time. A WSP should, therefore, maximize its average profit by 'optimally' (in some sense) (1) controlling the admission/rejection of arriving CR end-users, and (2) determining a policy of evicting in-service opportunistic users at the channel to which primary users return. To our best knowledge, this is the first attempt to jointly optimize admission and eviction controls for the dynamic spectrum market.

The WSP's profit maximization problem is formulated with a Semi-Markov Decision Process (SMDP) and its corresponding Linear Programming (LP) setup. This problem is found to become Nonlinear Programming (NLP) subject to two Quality-ofService $(\mathrm{QoS})$ constraints on request-blocking and user-dropping probabilities, which can fortunately be converted to LP via some manipulation. Using an extensive numerical analysis, we discovered that the derived optimal policy achieves up to $81 \%$ more profit than a Complete-Sharing (CS) algorithm in the tested scenario. We also investigate tradeoffs between the two QoS constraints, and consider other important factors affecting WSP's profit maximization such as the number of leased channels, the end-user pricing, and the cost for reimbursing evicted customers.
\end{abstract}

\section{INTRODUCTION}

Dynamic Spectrum Access (DSA) has opened a new way of solving the spectrum scarcity problem that is expected to occur due to the conventional static spectrum allocation policy as well as rapidly growing wireless applications and services. The static allocation policy has already reached its limits in finding unallocated spectrum for new emerging wireless services, since there has been an exponential growth of spectrum demand over the past decade or so [1]. DSA is expected to solve, or at least alleviate, this problem by enabling unlicensed secondary users (SUs) to opportunistically utilize the licensed spectrum whenever it is left unused by legacy primary users (PUs).

The application of DSA ranges from public to commercial and military networks. We will, however, focus on commercial networks, especially spectrum resource allocation to Cognitive
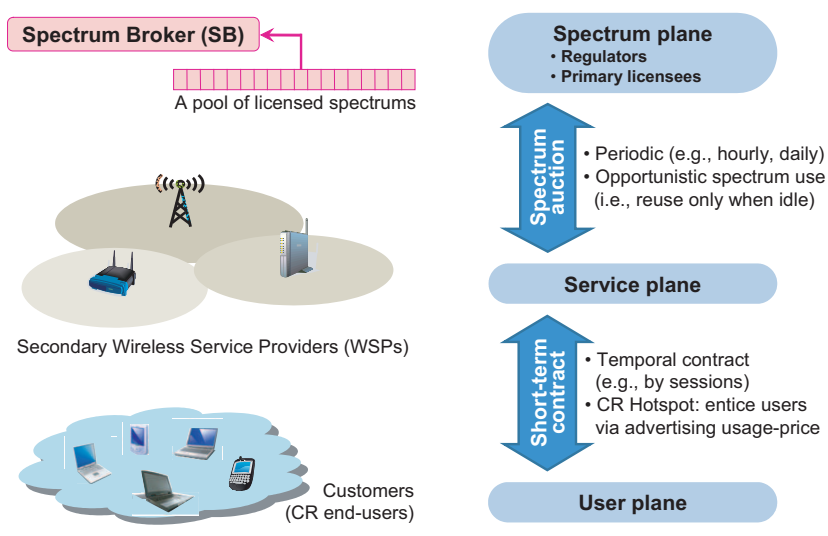

Fig. 1. Inter-plane interactions in the dynamic spectrum market

Radio (CR) users ${ }^{1}$ in such networks, since the benefit of DSA can be easily captured by widely spread commercial services.

In a commercial DSA network, the primary license holders may temporarily transfer their spectrum usage rights to $\mathrm{CR}$ users (i.e., SUs) [2], [3]. The Dynamic Spectrum Market (DSM) is a secondary spectrum market that facilitates the transfer of spectrum rights via an auction mechanism. DSM is composed of three interacting layers/planes as shown in Fig. 1: spectrum plane, service plane, and user plane [3], [4]. The spectrum is auctioned by a Spectrum Broker (SB) at the top plane where bidders are the secondary WSPs residing at the middle plane [5], [6]. Here, the SB might be either the regulatory authorities (e.g., FCC in USA and Ofcom in UK) or an authorized third-party. A secondary WSP leases spectrum via auction and subleases a portion of the leased channels to its customers at the bottom plane [7].

\section{A. Motivations}

1) Preemptable Spectrum Leasing: In [2], Buddhikot introduced four types of dynamic spectrum access-command and control, exclusive-use, shared-use, and commons. Of these, the following two types seem to prevail in the literature. First, in the shared-use model, SUs are opportunistically access the licensed bands without paying any usage charge to licensees. Their spectrum reuse is allowed as long as the SUs do not

\footnotetext{
${ }^{1}$ Throughout this paper, we will use the terms SUs and CR users interchangeably.
} 
interfere with the PUs. The IEEE 802.22 [8] is an example of such type where SUs opportunistically access TV bands. Although this model may achieve more efficient spectrum utilization, it is not likely to be accepted in the current market since there is no economic incentive for the license holders. Second, in the dynamic exclusive-use model, licensees can dynamically lease their spectrum to the lessee (i.e., SU) who temporarily owns an exclusive right to access the spectrum. Although this model can provide extra profit to the licensees, the PUs cannot re-claim their channels before the leasing period ends even if they encounter an unexpected shortage of spectrum.

We propose a new model which is a combination of the above two types by exploiting their advantages. In our model, the license holders can temporarily lease their channels to SUs and generate an additional revenue by charging for their opportunistic use of paid-but-idle channels. The SUs are allowed to use the leased channels only when they are not occupied by the PUs because the licensed users are given priority over the unlicensed CR users. However, the SUs can also benefit from this because they can access the spectrum at a much lower cost than legacy services. In this sense, our model can be considered as a preemptable secondary licensing of spectrum.

2) CR HotSpots Owned by Secondary WSPs: We view $C R$ HotSpots as a typical CR service provided by secondary WSPs in the commercial DSA scenario. They are akin to WLAN HotSpots located at popular public sites like coffee shops, stadiums, libraries, or airports, where a WSP owns CR access points and provides CR end-users wireless services, such as Internet access. The WSP advertises its pricing policy to entice customers, and each CR end-user sets up a short-term contract with the WSP. This type of service may emerge in early stages of DSA deployment due to its similarity to, and familiarity with, the wireless services available today.

3) Profit Maximization by secondary WSPs: This paper focuses on how a secondary WSP operating CR HotSpots can maximize its profit by user admission and eviction controls at each CR HotSpot when the spectrum demand varies with $\mathrm{CR}$ users and applications, e.g., audio/video users will need more bandwidth than text-only users. This particular application of DSA, in fact, reflects more general optimization issues in various DSA scenarios including public and military networks. The revenue and cost considered in this paper can be generalized as "reward," with which various types of goal can be expressed and optimized. For example, in the battlefield network, user-admission control may focus on maintaining reliable communication channels within the troops by assigning better quality channels to the commanding officers, where the reward is interpreted as link quality or available bandwidth.

Since the usage charges may differ between the users requesting different amounts of spectrum, accepting user arrivals whenever there is room in the leased channels, called Complete Sharing (CS) [9], does not necessarily maximize the WSP's profit. For example, it is sometimes more profitable for the WSP to reject a user's request with a small spectrum demand, even if there is room for it, and to wait until a user with a larger spectrum demand arrives. Thus, the optimal admission control is essential to the profit maximization.

A policy of evicting in-service users also plays an important role in maximizing profit due to PUs' ON-OFF channel usage patterns. Since PUs are given priority over SUs, the SUs must vacate a channel (called channel vacation) when its PUs return to the channel (i.e., the channel state changes from OFF to ON). At the time of channel vacation, the customers previously assigned to the channel have to be relocated by the WSP to the other remaining idle channels. However, if the remaining idle channels cannot fully support spectrum demands of all in-service users, ${ }^{2}$ the WSP should determine which users to be evicted from its network. The evicted users will be compensated with some form of reimbursement, which may differ by the user-specific spectrum demand and thus affect the WSP's profit.

\section{B. Contributions}

We address the problem of determining the optimal user admission and eviction controls using a Semi-Markov Decision Process (SMDP), since user arrivals/departures and channelstate transitions are stochastic. For SMDP, there are a number of well-known efficient algorithms to determine the optimal actions (i.e., admission or eviction) at each decision epoch. Using the basic components of SMDP, a Linear Programming (LP) algorithm is formulated and analyzed. To our best knowledge, this is the first attempt to jointly optimize admission and eviction controls with time-varying spectrum resources.

QoS provisioning for CR end-users is also considered by adding two constraints to the SMDP formulation: the probability of blocking newly-arriving users, and the probability of evicting/dropping in-service users. By making these two QoS metrics upper-bounded, the customers can get some form of QoS assurance and the WSP can strike a balance between profit maximization and customer satisfaction. Although the problem becomes Nonlinear Programming (NLP) by introducing the second QoS metric (i.e., the dropping probability), we show in Section IV that it can be converted to LP by properly manipulating the inequality constraint on the dropping probability.

\section{Related Work}

Mutlu et al. [10] studied the problem of maximizing a WSP's average profit. However, they focused only on optimizing the pricing policy without considering admission control which could further increase the WSP's profit. Moreover, they assumed that PUs and SUs can coexist in the same channel (thus unneeding eviction control) which is not always possible since the PUs are given priority over SUs in accessing the licensed channels. Ishibashi et al. [11] considered multi-homed PUs, where each PU is either classical or CR-enabled. They investigated enhancement of resource utilization with cognitive PUs switching between channels, and derived the blocking and

\footnotetext{
${ }^{2}$ We do not consider QoS degradation (i.e., assigning less bandwidth than a user wants) in this paper.
} 
dropping probabilities in such a scenario. However, no priority in channel access is given to the classical PUs, thus unaccounting for channel eviction. Wang et al. [12] proposed a primaryprioritized Markov approach where PUs have exclusive rights to access their own channels. Although the problem setup is somewhat similar to ours, they only considered a single channel and two SUs, thus limiting its applicability. Moreover, they did not consider admission control. Ross and Tsang [9] investigated the problem of optimal admission control when spectrum-demand varies with users. However, channels are assumed always available (i.e., always OFF), and hence, user eviction was not studied.

\section{Organization}

Section II introduces the system model and basic assumptions used in this paper. In Section III, the problem of maximizing a WSP's profit is modeled as SMDP and its relevant components are derived. Section IV presents an LP-based SMDP algorithm to determine the optimal user admission and eviction policy with two QoS constraints, blocking and dropping probabilities. The proposed scheme is evaluated in Section V via numerical analysis and simulation, and then the paper concludes with Section VI.

\section{SySTEM MOdEL}

\section{A. Channel Model}

As illustrated in Fig. 2, we model a channel as an ON/OFF alternating renewal process [13], denoted by $Z^{i}(t)(i$ : channel index). This type of channel model has been successfully used in many applications [14]-[16], where the ON (or OFF) period implies the duration with (or without) PUs' signal activities. Hence, SUs are allowed to access the channel only within OFF periods. We assume that ON/OFF transitions can be detected by spectrum sensing, which is not a main focus of this paper. Interested readers may refer to [16] for more details on spectrum sensing.

The sojourn times in ON and OFF states are represented by random variables $T_{O N}^{i}$ and $T_{O F F}^{i}$ whose probability density functions (pdfs) are denoted by $f_{T_{O N}^{i}}(t)$ and $f_{T_{O F F}^{i}}(t), t>0$, respectively. ON and OFF states are statistically independent of each other. Although $f_{T_{O N}^{i}}(t)$ and $f_{T_{O F F}^{i}}(t)$ can be any distribution function, here we consider exponentially-distributed ON and OFF periods with mean $1 / \mu_{O N}^{i}$ and $1 / \mu_{O F F}^{i}$, for mathematical tractability. Extension of our results to general $\mathrm{ON} / \mathrm{OFF}$ distributions is left as part of our future work.

Let $C_{i}$ denote the capacity (or bandwidth) of channel $i$. In this paper, we assume channel capacities are homogeneous, i.e., $C_{i}=C, \forall i$, for ease of illustration. However, our SMDP model can be easily extended to consider heterogeneous channel capacities at the expense of increased state/action spaces.

\section{B. Spectrum Auction Model}

We consider a multi-winner periodic spectrum auction [17], [18] where an SB (auctioneer) auctions off the licensed channels periodically (e.g., hourly, daily, or even weekly) every $T_{\text {auction }}$, and multiple WSPs bid for the number of channels
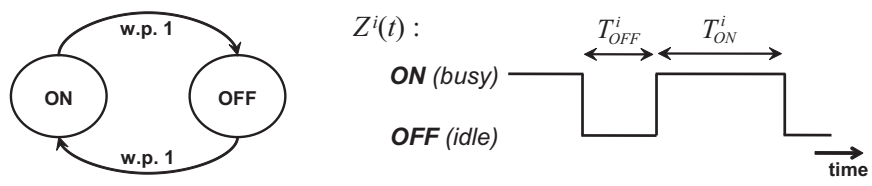

Fig. 2. Channel model: alternating renewal process with $\mathrm{ON}$ and $\mathrm{OFF}$ states

they want to lease. Once a WSP wins $M$ channels, it pays the (leasing) price $p_{b i d}(M)$ per unit-time to secure the temporal rights to reuse the $M$ channels for the period $T_{\text {auction }}$, and after $T_{\text {auction }}$ the leased channels are returned to the licensees.

We make the following assumption on the bidding cost function $p_{b i d}(M), p_{b i d}(0)=0$, which is often accepted in the literature on the CR auction market [7], [19]:

Assumption 1. $p_{b i d}(M)$ is a positive, non-decreasing and convex function of $M$.

This assumption is reasonable because the winning bid is likely to increase faster than proportionally to $M$ due to the competition between WSPs to acquire the limited amount of spectrum resources available in the market. The actual form of $p_{b i d}(M)$ should depend on the auction market, and hence we assume $p_{b i d}(M)$ is given a priori to focus on user-control issues. For an illustrative purpose, $p_{b i d}(M)=D_{1} \cdot M^{D_{2}}, D_{2} \geq$ 1, which is introduced in [7], [19] and satisfies Assumption 1 , will be used for simulation in Section $\mathrm{V}$ where $D_{2}$ implies the degree of competition in the auction.

With $M$ (possibly non-contiguous) leased channels, a WSP's total capacity becomes $M \cdot C$. Due to the ON/OFF channel usage patterns, however, its instantaneous capacity is given as $m \cdot C, 0 \leq m \leq M$, where $m$ is the number of idle channels at that instant. We assume that the pool of idle channels is managed as one logical channel, whose capacity is equal to $m \cdot C$ bandwidth-units. This is achievable via Orthogonal Frequency Division Multiplexing (OFDM) techniques with adaptive and selective allocation of OFDM sub-carriers, like NC-OFDM (Non-Contiguous OFDM) proposed in [20].

\section{Multi-class User QoS Model}

Customers are the CR-enabled end-devices seeking a secondary WSP that can best serve their needs. Each customer is assumed to have a spectrum demand which falls into one of the following $K$ QoS classes:

$$
\mathbf{B}=\left(B_{1}, B_{2}, \ldots, B_{K}\right)^{\mathrm{T}},
$$

where $B_{k}$ is the bandwidth requirement of class- $k$ customers ${ }^{3}$ and ${ }^{\mathrm{T}}$ represents 'transpose'. Without loss of generality, we can assume $B_{\text {min }}=B_{1}<B_{2}<\ldots<B_{K}=B_{\max }$.

Based on selective OFDM sub-carrier allocation, each CR end-user is assumed to be capable of tuning its antenna to any portion of the logical channel for its bandwidth assignment of $B_{k}$, as illustrated in Fig. 3. In this way, at a channel's $\mathrm{OFF} \rightarrow \mathrm{ON}$ transition, the users in the channel can be redistributed to other idle channels, by updating the mapping of

\footnotetext{
${ }^{3}$ We reserve $k$ and $i$ as class and channel indexes, respectively.
} 


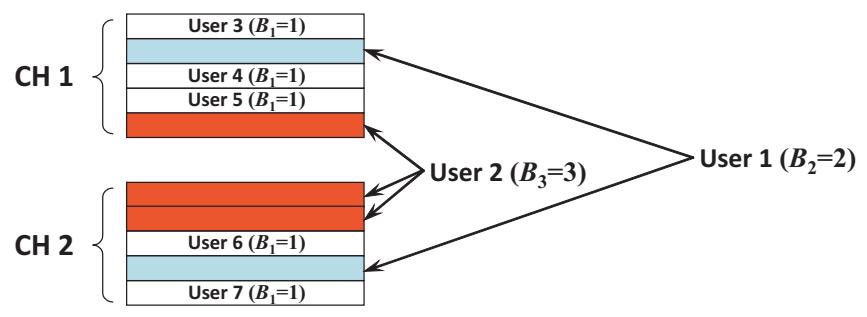

Fig. 3. An example of channel allocation with $M=2, K=3$, and $C=5$.

OFDM subcarriers to the users. MAC-layer beaconing might be used to perform this re-mapping in real time.

\section{User Traffic Model}

We assume that the arrival of class- $k$ customers follows a Poisson process with rate $\lambda_{k}$. As shown in [21], useroriented connection requests are modeled well as a Poisson arrival process. Since the requests from the CR users are connection-level, this assumption captures reasonably well the reality of the secondary market. For mathematical tractability, the service time of an admitted class- $k$ customer is assumed exponentially distributed with mean $1 / \mu_{k}$. This assumption captures some reality of wireless applications such as phonecall traffic with exponentially-distributed talk spurt and silence periods [22].

\section{E. End-User Pricing Model}

The revenue of a WSP is generated from the CR endusers who pay fees for their opportunistic spectrum usage. The vector of the usage fees for $K$ QoS-classes, in terms of price per unit-time per unit-bandwidth, is denoted as

$$
\mathbf{p}=\left(p_{1}, p_{2}, \ldots, p_{K}\right)^{\mathrm{T}},
$$

where $p_{k}$ could be either constant, non-decreasing, or nonincreasing in $k$, depending on the WSP's pricing policy.

The arrival rate is in fact price-dependent, and therefore, it is denoted by $\lambda_{k}\left(p_{k}\right) . \lambda_{k}\left(p_{k}\right)$ is a non-increasing function of $p_{k}$, since a WSP advertising higher prices should expect less customer arrivals than its competitors offering lower prices. The actual price-arrival relationship depends highly on the WSP's tariff and the degree of competition, which must be treated as a separate marketing research problem. Here we assume $\lambda_{k}\left(p_{k}\right)$ is given a priori and confine our discussion to optimal user control. We leave $\lambda_{k}\left(p_{k}\right)$ as a general parameter in the analysis, and we will use the following example in Section V for an illustrative purpose: $\lambda_{k}\left(p_{k}\right)=\lambda_{k}^{\max } e^{-\delta_{k} p_{k}}$, as introduced in [23]. Here, $\lambda_{k}^{\max }$ indicates that the maximum user population at a CR HotSpot is bounded, and $\delta_{k}$ represents the rate of decrease of the arrival rate as $p_{k}$ increases, which is related to the degree of competition between WSPs.

\section{SMDP FORMULATION}

The profit maximization at each CR HotSpot faces a unique challenge due to time-varying channel availability. Therefore, a secondary WSP must jointly consider user admission and eviction controls to maximize its profit subject to some QoS constraints, such as keeping the blocking and dropping probabilities within an acceptable range.

We formulate SMDP by accounting for time-varying channel availability. The basic and essential components of the SMDP are derived by considering possible actions to be taken for the joint control of user admission and eviction.

\section{A. System State and State Space}

We start with the definition of system state as

$$
\mathbf{s}=(\mathbf{n}, \mathbf{w}), \quad\left\{\begin{array}{l}
\mathbf{n}=\left(n_{1}, n_{2}, \ldots, n_{K}\right)^{\mathrm{T}} \\
\mathbf{w}=\left(w_{1}, w_{2}, \ldots, w_{M}\right)^{\mathrm{T}}
\end{array}\right.
$$

where $n_{k}$ is the number of class- $k$ customers in service, and $w_{i}$ is the channel state defined as:

$w_{i}=\left\{\begin{array}{l}1, \text { channel } i \text { is occupied by primaries (i.e., ON), } \\ 0, \text { channel } i \text { is not occupied by primaries (i.e., OFF). }\end{array}\right.$

The set of possible sub-states $\mathbf{n}$ for a given channel state $\mathbf{w}$ is thus determined as

$$
\Lambda_{\mathbf{n} \mid \mathbf{w}}=\left\{\mathbf{n}: n_{k} \geq 0, \quad \mathbf{n}^{\mathrm{T}} \mathbf{B} \leq m C, \quad m=(\mathbf{1}-\mathbf{w})^{\mathrm{T}} \mathbf{1}\right\},
$$

where $m$ is the number of idle channels as defined earlier, and 1 is a vector of 1's. In addition, the set of possible sub-states $\mathbf{w}$ is given as

$$
\Lambda_{\mathbf{w}}=\left\{\mathbf{w}: w_{i} \in\{0,1\}\right\} .
$$

Therefore, the state space $\Lambda$ is given as

$$
\Lambda=\left\{\mathbf{s}: \mathbf{w} \in \Lambda_{\mathbf{w}}, \mathbf{n} \in \Lambda_{\mathbf{n} \mid \mathbf{w}}\right\} .
$$

\section{B. Possible Actions and Action Space}

In our SMDP formulation, an action is taken and updated at each decision epoch under the chosen policy, where a natural choice of the decision epoch is the instant when system state changes, i.e., (1) at class- $k$ customer's arrival/departure and (2) at channel $i$ 's state transition ( $\mathrm{ON} \rightarrow \mathrm{OFF}$ or $\mathrm{OFF} \rightarrow \mathrm{ON}$ ).

We define the action at a certain decision epoch as

$$
\boldsymbol{\alpha}=(\mathbf{a}, \mathbf{b}), \quad\left\{\begin{array}{l}
\mathbf{a}=\left(a_{1}, a_{2}, \ldots, a_{K}\right)^{\mathrm{T}}, \\
\mathbf{b}=\left(b_{1}, b_{2}, \ldots, b_{K}\right)^{\mathrm{T}},
\end{array}\right.
$$

where $\mathbf{a}$ is the admission policy for future customer arrivals, such that

$$
a_{k}= \begin{cases}0, & \text { reject } \text { all future class- } k \text { arrivals, } \\ 1, & \text { admit all future class- } k \text { arrivals, }\end{cases}
$$

and $\mathbf{b}$ is the eviction policy of in-service customers with $b_{k}$ indicating the number of class- $k$ customers to be evicted at the time of channel vacation. Note that $\mathbf{b}$ is effective only when a channel vacation happens (i.e., at $\mathrm{OFF} \rightarrow \mathrm{ON}$ of a certain channel). That is, even if $\mathbf{b} \neq \mathbf{0}$, we do not evict any customer if the next event is arrival, departure, or $\mathrm{ON} \rightarrow \mathrm{OFF}$. 
Then, for a given state $\mathbf{s}$, the action space $A$ is defined as

$$
\begin{aligned}
& A_{1}(\mathbf{s})=\left\{\mathbf{a}: a_{k} \in\{0,1\} ; a_{k}=0 \text { if }\left(\mathbf{n}+\mathbf{u}_{k}\right)^{\mathrm{T}} \mathbf{B}>m C\right\}, \\
& A_{2}(\mathbf{s})=\left\{\begin{array}{l}
\mathbf{b}: 0 \leq b_{k} \leq n_{k}, \quad(\mathbf{n}-\mathbf{b})^{\mathrm{T}} \mathbf{B} \leq(m-1) C, \\
\left(\mathbf{n}-\mathbf{b}+\mathbf{u}_{k}\right)^{\mathrm{T}} \mathbf{B}>(m-1) C \text { for } \forall k \text { s.t. } b_{k} \neq 0 ; \\
\left.\mathbf{b}=\mathbf{0} \text { if } \mathbf{n}^{\mathrm{T}} \mathbf{B} \leq(m-1) C\right\},
\end{array}\right. \\
& A(\mathbf{s})=\left\{\begin{array}{l}
\{\boldsymbol{\alpha}: \mathbf{a}=\mathbf{0}, \mathbf{b}=\mathbf{0}\}, \quad \text { if } m=0, \\
\left\{\boldsymbol{\alpha}: \mathbf{a} \in A_{1}(\mathbf{s}), \mathbf{b} \in A_{2}(\mathbf{s})\right\}, \text { otherwise, }
\end{array}\right.
\end{aligned}
$$

where $\mathbf{u}_{j}$ is a unit vector with a single 1 at the $j$-th position and 0's elsewhere. In the definition of $A_{2}(\mathbf{s})$, the constraint

$$
\left(\mathbf{n}-\mathbf{b}+\mathbf{u}_{k}\right)^{\mathrm{T}} \mathbf{B}>(m-1) C \text { for } \forall k \text { s.t. } b_{k} \neq 0
$$

implies that $\mathbf{b}$ should be minimal so as not to evict more inservice customers than necessary. That is, $\mathbf{b}$ is not minimal if there exists $k$ such that $\mathbf{b}^{\prime}=\mathbf{b}-\mathbf{u}_{k}$ and $\left(\mathbf{n}-\mathbf{b}^{\prime}\right)^{\mathrm{T}} \mathbf{B} \leq$ $(m-1) C$, i.e., evicting one less customer than $\mathbf{b}$ still fits in $(m-1)$ channels. Obviously, the choice of minimal $\mathbf{b}$ for a given $\mathbf{s}$ is not unique. For example, in case $m=2, C=5$ and $\mathbf{B}=(1,2)$, there are three possible minimal $\mathbf{b}$ 's for $\mathbf{n}=$ $(5,2): \mathbf{b}=(4,0), \mathbf{b}=(2,1)$ and $\mathbf{b}=(0,2)$.

\section{Eligibility for SMDP Formulation}

Before deriving other essential components of the SMDP, we need to check if the system under consideration can be modeled as SMDP. In [24], SMDP is defined as follows.

Definition 1. A dynamic system is said to be a semi-Markov decision process if the following property is satisfied: if at a decision epoch the action $\alpha$ is chosen in state $s$, then the time until, the state at, and revenue/cost incurred until the next decision epoch depend only on the current state $s$ and its chosen action $\boldsymbol{\alpha}$.

According to our assumption, inter-arrival and interdeparture times of class- $k$ customers are all exponentially distributed. Since the arrival and departure processes are independent of each other, the combined random process is still exponentially distributed with mean $1 /\left(\mathbf{n}^{\mathrm{T}} \boldsymbol{\mu}+\mathbf{a}^{\mathrm{T}} \boldsymbol{\lambda}\right)$, where $\boldsymbol{\lambda}=\left(\lambda_{1}, \lambda_{2}, \ldots, \lambda_{K}\right)^{\mathrm{T}}$, and $\boldsymbol{\mu}=\left(\mu_{1}, \mu_{2}, \ldots, \mu_{K}\right)^{\mathrm{T}}$. Second, since channel $i$ 's residual time in $\mathrm{ON}$ (or OFF) state is exponentially distributed with mean $1 / \mu_{O N}^{i}$ (or $1 / \mu_{O N}^{i}$ ), channel $i$ 's state transition process becomes exponentially distributed with mean $1 /\left(\left(1-w_{i}\right) \mu_{O F F}^{i}+w_{i} \mu_{O N}^{i}\right)$. Therefore, with $M$ independent channels, the combined random process is also exponentially distributed with mean $\left[(\mathbf{1}-\mathbf{w})^{\mathrm{T}} \boldsymbol{\mu}_{\boldsymbol{O F F}}+\mathbf{w}^{\mathrm{T}} \boldsymbol{\mu}_{\boldsymbol{O N}}\right]^{-1}$, where $\boldsymbol{\mu}_{\boldsymbol{O F F}}=\left(\mu_{O F F}^{1}, \mu_{O F F}^{2}, \ldots, \mu_{O F F}^{M}\right)^{\mathrm{T}}$ and $\boldsymbol{\mu}_{\boldsymbol{O N}}=$ $\left(\mu_{O N}^{1}, \mu_{O N}^{2}, \ldots, \mu_{O N}^{M}\right)^{\mathrm{T}}$. Finally, the combination of all four possible events-arrival and departure (class- $k$ ), and $\mathrm{ON} \rightarrow \mathrm{OFF}$ and $\mathrm{OFF} \rightarrow \mathrm{ON}$ transitions (channel $i$ )-becomes exponentially distributed with mean

$$
\left[\mathbf{n}^{\mathrm{T}} \boldsymbol{\mu}+\mathbf{a}^{\mathrm{T}} \boldsymbol{\lambda}+(\mathbf{1}-\mathbf{w})^{\mathrm{T}} \boldsymbol{\mu}_{\boldsymbol{O F F}}+\mathbf{w}^{\mathrm{T}} \boldsymbol{\mu}_{\boldsymbol{O} \boldsymbol{N}}\right]^{-1} .
$$

As a result, the system model considered in this paper can be modeled as SMDP, since it possesses the necessary properties in Definition 1 thanks to the memoryless property of an exponential distribution.

\section{Decision Epochs}

Since the combined process of four possible events is exponentially distributed with mean as in Eq. (1), the expected time between two decision epochs, denoted by $\tau_{\mathbf{s}}(\boldsymbol{\alpha})$, is determined as

$$
\tau_{\mathbf{s}}(\boldsymbol{\alpha})=\left[\mathbf{n}^{\mathrm{T}} \boldsymbol{\mu}+\mathbf{a}^{\mathrm{T}} \boldsymbol{\lambda}+(\mathbf{1}-\mathbf{w})^{\mathrm{T}} \boldsymbol{\mu}_{\boldsymbol{O F F}}+\mathbf{w}^{\mathrm{T}} \boldsymbol{\mu}_{\boldsymbol{O N}}\right]^{-1} .
$$

\section{E. State-Transition Probability}

The probability that the state of the system switches from $\mathbf{s}=(\mathbf{n}, \mathbf{w})$ to $\mathbf{s}^{\prime}=\left(\mathbf{n}^{\prime}, \mathbf{w}^{\prime}\right)$ at the next decision epoch is

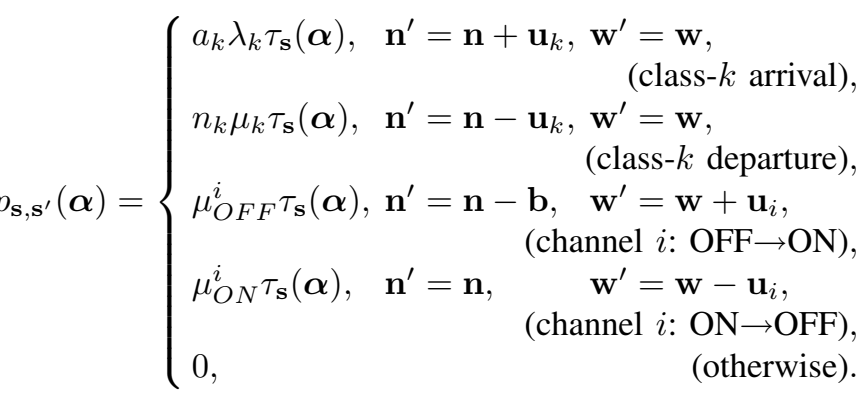

\section{F. Revenue and Reimbursement Cost}

Let $r_{\mathbf{s}}(\boldsymbol{\alpha})$ and $c_{\mathbf{s}}(\boldsymbol{\alpha})$ denote the expected revenue and the cost incurred by customers until the next decision epoch if action $\boldsymbol{\alpha}$ is chosen at state $\mathbf{s}$, respectively. Since the revenue comes from the usage fee paid by the admitted customers, $r_{\mathbf{s}}(\boldsymbol{\alpha})$ is given as

$$
r_{\mathbf{s}}(\boldsymbol{\alpha})=\sum_{k} p_{k} B_{k} n_{k} \tau_{\mathbf{s}}(\boldsymbol{\alpha}) .
$$

Assuming a fixed amount of reimbursement $I_{k}$ for an evicted class- $k$ customer, $c_{\mathbf{s}}(\boldsymbol{\alpha})$ is

$$
\begin{aligned}
c_{\mathbf{s}}(\boldsymbol{\alpha}) & =\sum_{k} I_{k} b_{k} \cdot q_{\mathbf{s}}^{\mathcal{V}}(\boldsymbol{\alpha}), \\
q_{\mathbf{s}}^{\mathcal{V}}(\boldsymbol{\alpha}) & \sum_{\substack{\mathbf{s}^{\prime} \in \Lambda \text { s.t. } \mathbf{n}^{\prime}=\mathbf{n}-\mathbf{b}, \mathbf{w}^{\prime}=\mathbf{w}+\mathbf{u}_{i} \text { for some } i}} p_{\mathbf{s}, \mathbf{s}^{\prime}}(\boldsymbol{\alpha}),
\end{aligned}
$$

where $\tau_{\mathbf{s}}(\boldsymbol{\alpha})$ does not contribute to the equation since the reimbursement is a one-time cost at channel vacation. $q_{\mathbf{s}}^{\mathcal{V}}(\boldsymbol{\alpha})$ in the above equation implies the probability that the event of channel vacation will happen at the next decision epoch. We also let $q_{\mathbf{s}}^{\mathcal{D}}(\boldsymbol{\alpha})$ denote the probability that the event of class $k$ departure will happen at the next decision epoch, which is defined as:

$$
q_{\mathbf{s}}^{\mathcal{D}}(\boldsymbol{\alpha})=\sum_{\substack{\mathbf{s}^{\prime} \in \Lambda \text { s.t. } \\ \mathbf{n}^{\prime}=\mathbf{n}-\mathbf{u}_{k}, \mathbf{w}^{\prime}=\mathbf{w}}} p_{\mathbf{s}, \mathbf{s}^{\prime}}(\boldsymbol{\alpha})
$$

\section{Optimal User Control via An SMDP Algorithm}

So far we have defined and derived all necessary components of SMDP. Now, we want to find the optimal action $\boldsymbol{\alpha}$ for each possible system state $\mathbf{s}$ to maximize the average profit, using the derived SMDP components. There are three wellknown methods for optimally solving the SMDP problem: policy-iteration, value-iteration, and Linear Programming (LP) 
[24]. Of these, LP is the best known method to model the QoS-constrained optimization problem thanks to its flexibility to include additional equality and inequality constraints. Therefore, we formulate an LP problem for the given SMDP with the two QoS-constraints.

\section{A. Linear Programming SMDP Algorithm: Constrained QoS}

Here we formulate a three-step LP algorithm with the two given QoS constraints: the probability of blocking class- $k$ arrivals (denoted by $P_{b}^{k}$ ), and the probability of evicting class- $k$ in-service customers (denoted by $P_{d}^{k}$ ). This 3-step algorithm follows the general format of the LP algorithm recommended in [24], but its mathematical contents are our own development.

Step 1: Find the optimal basic solution $z_{\mathrm{s}, \alpha}^{*}$ to the following linear programming $\left(z_{\mathbf{s}, \boldsymbol{\alpha}} \geq 0\right)$ :

Maximize $\sum_{\mathbf{s} \in \Lambda} \sum_{\boldsymbol{\alpha} \in A(\mathbf{s})}\left(r_{\mathbf{s}}(\boldsymbol{\alpha})-c_{\mathbf{s}}(\boldsymbol{\alpha})\right) z_{\mathbf{s}, \boldsymbol{\alpha}}$

\section{Subject to}

$$
\begin{aligned}
\sum_{\boldsymbol{\alpha} \in A\left(\mathbf{s}^{\prime}\right)} z_{\mathbf{s}^{\prime}, \boldsymbol{\alpha}}-\sum_{\mathbf{s} \in \Lambda} \sum_{\boldsymbol{\alpha} \in A(\mathbf{s})} p_{\mathbf{s}, \mathbf{s}^{\prime}}(\boldsymbol{\alpha}) z_{\mathbf{s}, \boldsymbol{\alpha}} & =0, \quad \mathbf{s}^{\prime} \in \Lambda, \\
\sum_{\mathbf{s} \in \Lambda} \sum_{\boldsymbol{\alpha} \in A(\mathbf{s})} \tau_{\mathbf{s}}(\boldsymbol{\alpha}) z_{\mathbf{s}, \boldsymbol{\alpha}} & =1, \\
P_{b}^{k} & \leq \gamma_{\text {block }}^{k}, \quad \forall k, \\
P_{d}^{k} & \leq \gamma_{d r o p}^{k}, \quad \forall k,
\end{aligned}
$$

where $\gamma_{b l o c k}^{k}$ and $\gamma_{d r o p}^{k}$ are the upper-bounds on $P_{b}^{k}$ and $P_{d}^{k}$. In addition, $P_{b}^{k}$ and $P_{d}^{k}$ are expressed as follows:

$$
\begin{aligned}
& P_{b}^{k}=\sum_{\mathbf{s} \in \Lambda} \sum_{\substack{\boldsymbol{\alpha} \in A(\mathbf{s}) \\
\text { s.t. } a_{k}=0}} \tau_{\mathbf{s}}(\boldsymbol{\alpha}) z_{\mathbf{s}, \boldsymbol{\alpha}}, \\
& P_{d}^{k}=\frac{\mathcal{V}}{\mathcal{D}+\mathcal{V}},\left\{\begin{array}{l}
\mathcal{D}=\sum_{\mathbf{s} \in \Lambda} \sum_{\boldsymbol{\alpha} \in A(\mathbf{s})} \tau_{\mathbf{s}}(\boldsymbol{\alpha}) z_{\mathbf{s}, \boldsymbol{\alpha}} q_{\mathbf{s}}^{\mathcal{D}}(\boldsymbol{\alpha}) \cdot 1, \\
\mathcal{V}=\sum_{\mathbf{s} \in \Lambda} \sum_{\boldsymbol{\alpha} \in A(\mathbf{s})} \tau_{\mathbf{s}}(\boldsymbol{\alpha}) z_{\mathbf{s}, \boldsymbol{\alpha}} q_{\mathbf{s}}^{\mathcal{V}}(\boldsymbol{\alpha}) \cdot b_{k},
\end{array}\right.
\end{aligned}
$$

where $z_{\mathbf{s}, \boldsymbol{\alpha}}=x_{\mathbf{s}, \boldsymbol{\alpha}} / \tau_{\mathbf{s}}(\boldsymbol{\alpha})$, and $x_{\mathbf{s}, \boldsymbol{\alpha}}$ denotes the fraction of time that the system is in state $\mathbf{s}$ when action $\boldsymbol{\alpha}$ is chosen. Therefore, $\mathcal{D}$ implies the expected number of departure until the next decision epoch and $\mathcal{V}$ implies the expected number of evictions until the next decision epoch.

Due to the form of $P_{d}^{k}$, this becomes a Nonlinear Programming (NLP) problem. Fortunately, however, by properly manipulating the constraint $P_{d}^{k} \leq \gamma_{d r o p}^{k}$, it can be converted to a Linear Programming (LP) problem as follows:

$$
\begin{aligned}
& P_{d}^{k}=\frac{\mathcal{V}}{\mathcal{D}+\mathcal{V}} \leq \gamma_{d r o p}^{k} \Rightarrow\left(1 / \gamma_{d r o p}^{k}-1\right) \mathcal{V} \leq \mathcal{D} \Rightarrow \\
& \sum_{\mathbf{s} \in \Lambda} \sum_{\boldsymbol{\alpha} \in A(\mathbf{s})}\left(q_{\mathbf{s}}^{\mathcal{V}}(\boldsymbol{\alpha}) \frac{1-\gamma_{d r o p}^{k}}{\gamma_{d r o p}^{k}} \cdot b_{k}-q_{\mathbf{s}}^{\mathcal{D}}(\boldsymbol{\alpha})\right) \tau_{\mathbf{s}}(\boldsymbol{\alpha}) z_{\mathbf{s}, \boldsymbol{\alpha}} \leq 0,
\end{aligned}
$$

which is a linear constraint on $z_{\mathbf{s}, \alpha}$ 's.
Step 2: Start with a non-empty set

$$
S:=\left\{\mathbf{s} \mid \sum_{\boldsymbol{\alpha} \in A(\mathbf{s})} z_{\mathbf{s}, \boldsymbol{\alpha}}^{*}>0\right\},
$$

and for any state $\mathbf{s} \in S$, set the decision as

$$
R^{*}(\mathbf{s}):=\boldsymbol{\alpha} \text { for some } \boldsymbol{\alpha} \text { such that } z_{\mathbf{s}, \boldsymbol{\alpha}}^{*}>0 .
$$

Step 3: If $S=\Lambda$, then the algorithm terminates with the optimal policy $R^{*}$. Otherwise, determine some state $\mathbf{s} \notin S$ and action $\boldsymbol{\alpha} \in A(\mathbf{s})$ such that $p_{\mathbf{s}, \mathbf{s}^{\prime}}(\boldsymbol{\alpha})>0$ for some $\mathbf{s}^{\prime} \in S$. For the chosen $\mathbf{s}$, set $R^{*}(\mathbf{s}):=\alpha$ and update $S:=S \cup\{\mathbf{s}\}$, and then repeat Step 3.

By repeatedly performing Step 3, the algorithm runs until $S$ becomes $\Lambda$. The complexity of executing this final step is trivial since $p_{\mathbf{s}, \mathbf{s}^{\prime}}(\boldsymbol{\alpha})>0$ has already been computed in Step 1 for all possible combinations of $\left(\mathbf{s}, \mathbf{s}^{\prime}, \boldsymbol{\alpha}\right)$. In addition, the optimality of the derived policy is guaranteed in [24], although the algorithm may not produce a unique solution due to the conditions 'some $\boldsymbol{\alpha}$ ' and 'some $\mathrm{s}^{\prime}$ ' in step 2 and 3.

Then, the optimal profit $g^{*}$ per unit-time is determined as

$$
g^{*}=\sum_{\substack{\mathbf{s} \in \Lambda \\ \boldsymbol{\alpha}=R^{*}(\mathbf{s})}}\left(r_{\mathbf{s}}(\boldsymbol{\alpha})-c_{\mathbf{s}}(\boldsymbol{\alpha})\right) z_{\mathbf{s}, \boldsymbol{\alpha}}-p_{b i d}(M) .
$$

\section{B. Convergence of the SMDP algorithm}

The convergence of the above algorithm must be shown to ensure that it completes in a finite number of steps. Its convergence can be proven by showing that the considered MDP is unichain [25], where "unichain" is defined as follows.

Definition 2. An MDP is unichain if it consists of a single recurrent class plus a (possibly empty) set of transient states for every stationary policy $R$. [25]

Definition 3. A recurrent class is a communication class such that no state outside the class is accessible from any state inside it [26].

We now prove that the MDP considered here is unichain.

Proposition 1. The SMDP considered here is unichain, and thus the proposed LP algorithm converges to the optimal stationary policy in a finite number of steps.

Proof: Suppose there exist more than one recurrent class, and assume the state $\mathbf{s}_{0}=(\mathbf{0}, \mathbf{1})$ is in a recurrent class $S_{0}$. Then, for any $\mathbf{s} \notin S_{0}, \mathbf{s}_{0}$ should not be accessible from $\mathbf{s}$ by Definition 3. However, $\mathbf{s}_{0}$ is in fact accessible from $\mathbf{s}$ within $m=(\mathbf{1}-\mathbf{w})^{\mathrm{T}} \mathbf{1} \leq M$ transitions with probability $p_{\mathbf{s}, \mathbf{s}^{(1)}} p_{\mathbf{s}^{(1)}, \mathbf{s}^{(2)}} \cdots p_{\mathbf{s}^{(m-1)}, \mathbf{s}_{0}}>0$, if we choose the states $\left\{\mathbf{s}^{(1)}, \mathbf{s}^{(2)}, \ldots, \mathbf{s}^{(m-1)}\right\}$ such that each transition makes one of the $m$ idle channels occupied while already occupied channels remain occupied. This is a contradiction. Therefore, there exists only one recurrent class, and the SMDP is thus unichain. 


\section{Complexity of SMDP Algorithm}

The complexity of the proposed algorithm is measured by the size of the search space $\Lambda \times A(\mathbf{s})$. One can easily see that $|\Lambda \times A(\mathbf{s})|$ increases exponentially as $M$ or $K$ increases, because $\left|\Lambda_{\mathbf{w}}\right|=2^{M}$ and $\left|A_{1}(\mathbf{s})\right|=2^{K}$ in the worst case. Note, however, that the LP can be solved off-line before a WSP starts a new spectrum leasing period (i.e., $T_{\text {auction }}$ ), so the derived optimal control can be stored in a database. Using the database, the WSP can perform user admission control in real time by simply looking up the database at every user arrival/departure or channel ON/OFF transition.

\section{Performance Evaluation}

In this section, the performance of the proposed SMDP algorithm is evaluated, and its sensitivity to $M$, p, and $I_{k}$ (reimbursement cost) is studied via a series of numerical analyses and simulations.

First, Section V-A presents the state transition diagram of the proposed SMDP algorithm and compares its optimal profit with the profit by the CS (Complete Sharing) algorithm [9]. Then, in Section V-B, we discuss how the blocking and dropping probabilities affect each other. In Section V-C, we study how sensitive the optimal profit is to the end-user pricing p. Finally, Section V-D discusses the relationship between the reimbursement cost and the optimal profit.

In all tests, simulation is performed by randomly generating Poisson arrivals/departures (as assumed in Section II) and exponentially-distributed ON/OFF durations. Each decision epoch takes an action in accordance with the set of optimal actions determined by the analysis. A simulation ran for 3,000 time units and the same simulation repeats 10 times to get its average performance.

The simulation parameters are set as follows.

1) Parameters common to all tests are

- $C=5$, and $B_{k}=k, \forall k ; p_{b i d}(M)=\frac{1}{15} M^{2.5}$,

- $E\left[T_{O F F}^{i}\right]=1, E\left[T_{O N}^{i}\right]=0.5, \forall i$,

- $\lambda_{k}\left(p_{k}\right)=\lambda_{k}^{\max } e^{-p_{k}}$, where $\left(\lambda_{1}^{\max }, \lambda_{2}^{\max }, \lambda_{3}^{\max }\right)=$ $\left(\frac{1}{4}, 1, \frac{3}{4}\right)$

- $I_{k}=\epsilon_{I} \times p_{k} B_{k} / \mu_{k}, 0 \leq \epsilon_{I} \leq 1$,

where $T_{O F F}^{i}$ and $T_{O N}^{i}$ are all exponentially distributed, and $I_{k}$ is set to be $\epsilon_{I} \cdot 100 \%$ of the average usage charge until departure (not eviction) for each class- $k$ user.

2) Test-specific parameters are shown in Table I, where $\gamma_{\text {block }}=\gamma_{\text {block }}^{k}$ and $\gamma_{\text {drop }}=\gamma_{\text {drop }}^{k}$ for all $k$. In addition, the end-user price $\mathbf{p}$, except in Section $\mathrm{V}-\mathrm{C}$, is given as

$$
\text { - } p_{k}=2 \times 0.7^{k-1} \text {, }
$$

so that $(k+1)$-th class users get $30 \%$ discounted per unitbandwidth price compared to the $k$-th class users. This type of pricing is frequently used in the actual market where customers using higher quality service (i.e., larger $B_{k}$ ), hence paying more usage fee of $p_{k} \cdot B_{k}$, are offered a discounted price. In Section V-C, $p_{k}$ will be defined separately to show the impact of varying prices on $g^{*}$.

\begin{tabular}{|c|c|c|c|c|c|c|}
\hline Section & $M$ & $K$ & $\gamma_{\text {block }}$ & $\gamma_{\text {drop }}$ & $\boldsymbol{\mu}$ & $\epsilon_{I}$ \\
\hline \hline V-A1 & 2 & 2 & 1.0 & 1.0 & $\left(1, \frac{1}{2}\right)$ & 0.5 \\
\hline V-A2 & $1-5$ & 3 & 1.0 & 1.0 & $\left(1, \frac{1}{2}, \frac{1}{4}\right)$ & 0.5 \\
\hline V-B (a) & 2 & 3 & 1.0 & $0.37-0.46$ & $\left(\frac{1}{2}, \frac{1}{2}, \frac{1}{2}\right)$ & 0.5 \\
\hline V-B (b) & 2 & 3 & $0.24-0.33$ & 1.0 & $\left(\frac{1}{2}, \frac{1}{2}, \frac{1}{2}\right)$ & 0.5 \\
\hline V-C & 2 & 2 & 0.45 & 0.45 & $\left(1, \frac{1}{2}\right)$ & 0.5 \\
\hline V-D & 2 & 2 & 0.45 & 0.45 & $\left(1, \frac{1}{2}\right)$ & $0.1-1.0$ \\
\hline
\end{tabular}

TABLE I

THE LIST OF TEST-SPECIFIC PARAMETERS

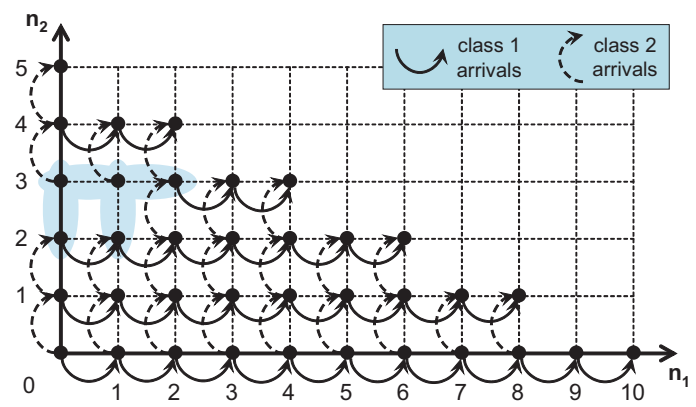

Fig. 4. The state-transition diagrams of the proposed SMDP algorithm, according to user arrivals (shown for $m=2$ )

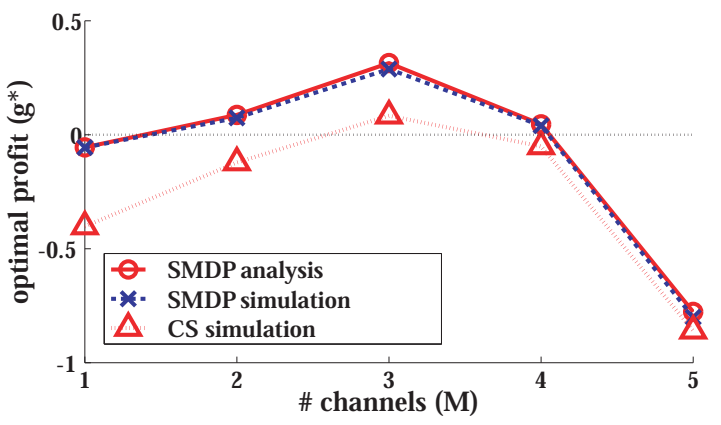

Fig. 5. Comparison of the profits achieved by SMDP and CS

\section{A. Optimal Control and Profit by the Proposed Algorithm}

1) State Transition Diagram: Fig. 4 illustrates the optimal actions derived by the proposed SMDP algorithm for $M=2$ and $K=2$, in the form of a state transition diagram. For simplicity, the state transition diagram is drawn for $m=2$ (i.e., when there are two idle channels) and only the transitions by the user arrivals are presented. The first thing we notice is that the optimal admission policy deliberately rejects some arrivals to maximize profit, and hence the following state transitions do not occur while they are allowed by the CS algorithm: $(0,2) \rightarrow(0,3),(0,3) \rightarrow(1,3),(1,2) \rightarrow(1,3)$, and $(1,3) \rightarrow(2,3)$. Second, it is known that if the $M$ channels are always available, the optimal admission policy for $K=2$ shows the threshold behavior [27]. However, we consider time-varying spectrum resource according to ON/OFF channel patterns, and interestingly it turns out that the optimal policy in this case does not follow the threshold behavior. This unique feature is observed partly because the states of $m=2$ drawn in Fig. 4 can make transition not only within the states of $m=2$ but also to the states of $m=1$ when channel vacation occurs in either of the two channels. 


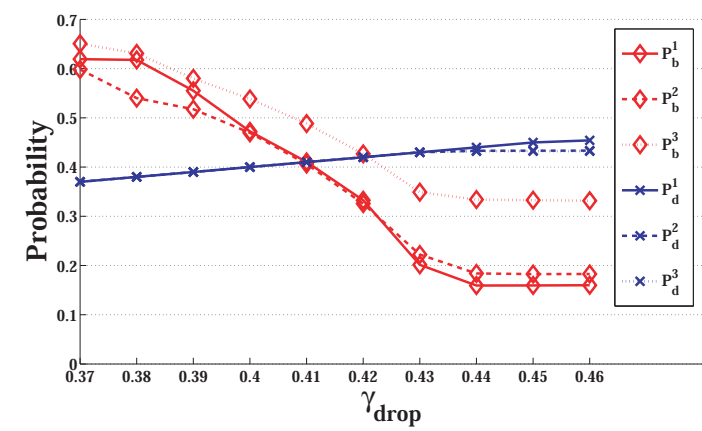

(a) Varying $\gamma_{d r o p}$

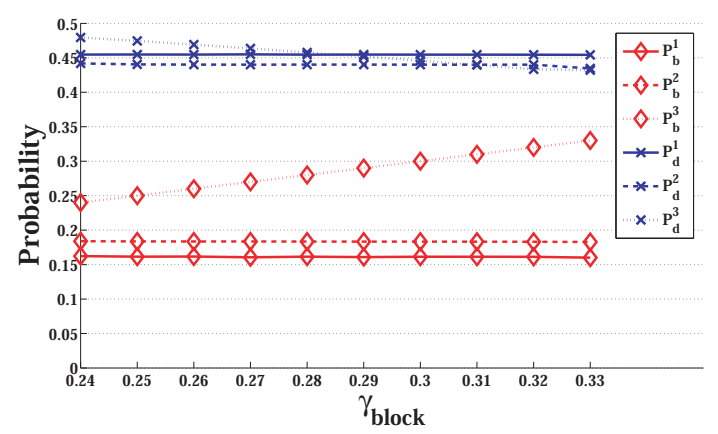

(b) Varying $\gamma_{b l o c k}$

Fig. 6. The tradeoffs between $P_{b}$ and $P_{d}$

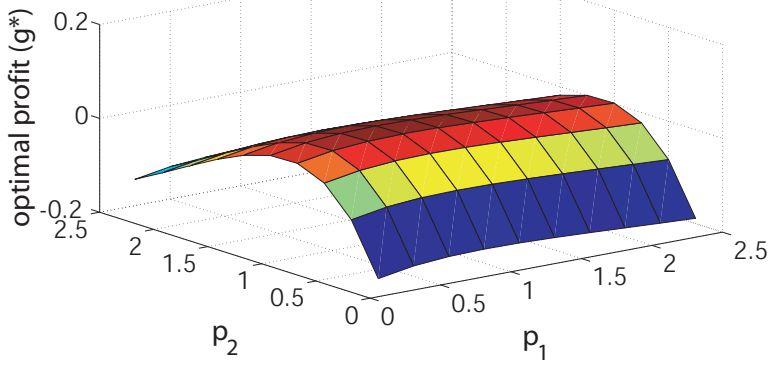

Fig. 7. Optimal profit with various end-user pricing

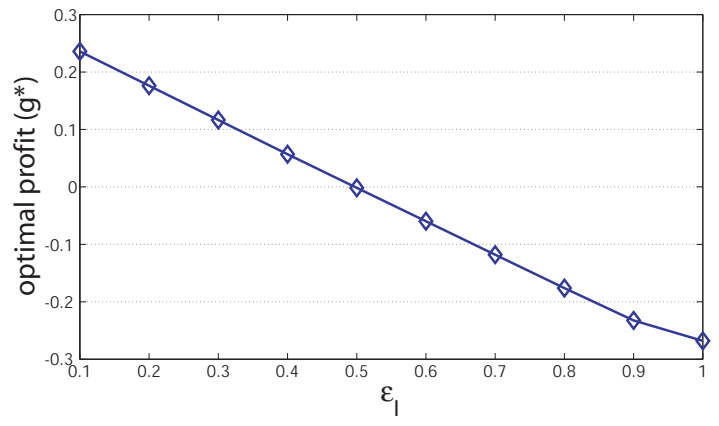

Fig. 8. Optimal profit with different reimbursement cost
2) Optimal Profit $g^{*}$ : We compare the performance of the proposed SMDP algorithm with the CS algorithm, in terms of $g^{*}$. In general, CS cannot achieve the maximal profit because it does not differentiate users with different spectrum demands. Moreover, the CS algorithm only provides a passive admission control with which a user is rejected only when there is no room in the WSP's resources. Since the traditional CS algorithm is not designed to deal with user evictions, we assume that it randomly chooses users to evict so that the remaining users can be packed into the idle channels. Since CS does not support any QoS guarantee, we use $\gamma_{\text {block }}=\gamma_{\text {drop }}=1.0$ for fair comparison.

Fig. 5 shows that SMDP always achieves more profit than CS. For SMDP, the simulation results conform to the analytically-predicted $g^{*}$ from Eq. (2), indicating that the proposed SMDP model in Section IV was properly analyzed and applied. One can also see that $g^{*}$ varies dramatically as $M$ changes. For example, $g^{*}$ is increasing when $M \leq 3$ and decreasing when $M \geq 3$. Interestingly, sometimes $g^{*}$ even becomes negative. This phenomenon stems from the tradeoff between the revenue generated by customers (i.e., $\left.g^{*}+p_{b i d}(M)\right)$ and the bidding cost (i.e., $p_{b i d}(M)$ ) because(1) a larger $M$ induces more revenue and less reimbursement cost due to more room available to accommodate user arrivals, but (2) the gain will eventually be saturated due to the bounded user population, and therefore, leasing more channels than necessary becomes unproductive, considering the fact that $p_{b i d}(M)$ grows faster than proportionally to $M$. Therefore, finding a proper $M$ is essential to maximizing the profit of a WSP. Since our proposed method is to achieve maximal profit $g^{*}$ at a given $M$, a WSP may use our method to find $g^{*}$ for each $M$ and then to determine the proper number of channels to bid (i.e., $M$ ) for maximal $g^{*}$.

\section{B. Tradeoffs Between Two QoS Constraints}

Fig. 6 plots the tradeoff between $P_{b}$ and $P_{d}$. To show the relationship between the two, we first fix $\gamma_{\text {block }}=1.0$ and vary $\gamma_{d r o p}$ in (a). Similarly, in (b), we fix $\gamma_{d r o p}=1.0$ and vary $\gamma_{b l o c k}$.

As $\gamma_{d r o p}$ decreases, the QoS requirement on $P_{d}$ becomes stricter in such a way that more in-service users should be protected from eviction at channel vacation. For this, user arrivals must be blocked more often since the longer stay of in-service users implies less idle resources for new arrivals. As a result, $P_{b}$ increases to compensate for the decrease of $P_{d}$, which can be observed from Fig. 6(a).

By contrast, as $\gamma_{b l o c k}$ decreases, the QoS requirement on $P_{b}$ becomes stricter so that less users are rejected upon their arrival. To accommodate more users, the WSP would have to reserve more room by evicting more users at channel vacation, and thus increasing $P_{d}$. However, as seen in Fig. 6(b), there is only a slight increase in $P_{d}$ as $\gamma_{b l o c k}$ decreases. This is rooted at the fact that an action $\mathbf{b}$ is chosen to be "minimal," and hence no more users than necessary are evicted. One can make $P_{d}$ more sensitive to $\gamma_{\text {block }}$ by introducing non-minimal b's in $A_{2}(\mathbf{s})$ at the cost of an enlarged action space. 


\section{Impact of End-User Pricing on $g^{*}$}

Due to the price-dependent arrival rate, i.e., $\lambda_{k}\left(p_{k}\right)=$ $\lambda_{k}^{\max } e^{-p_{k}}$, varying $p_{k}$ would produce different profits. To show its impact on $g^{*}$, we tested the case of $M=2$ and $K=2$, while varying $p_{1}$ and $p_{2}$ such as $0.25 \leq p_{1}, p_{2} \leq 2.50$. Unlike other tests, all possible combinations of $\left(p_{1}, p_{2}\right)$ are considered. Fig. 7 plots the resulting $g^{*}$. It can be seen that as $p_{k}$ gets closer to 0.25 and 2.50 , the resultant profit decreases due to the bounded user arrival by $\lambda_{k}^{\max }$ and decrease of the user arrival rate by $e^{-p_{k}}$, respectively. As a result, the profit function becomes concave, where the largest profit can be attained at $p_{1}=1.0, p_{2}=1.0$ in the figure. With the tested scenario, profit change is more sensitive to $p_{2}$ because $\lambda_{2}^{\max }>\lambda_{1}^{\max }$ and $1 / \mu_{2}>1 / \mu_{1}$. Therefore, a WSP must consider the impact of its pricing policy on the overall profit, for which marketing research is essential to discover the pricearrival rate relationship.

\section{Impact of Reimbursement on $g^{*}$}

Fig. 8 presents the impact of reimbursement policy on $g^{*}$, where $\epsilon_{I}$ determines how much of the usage charge should be provided for evicted users as a compensation. The plot clearly shows how sensitive the optimal profit is to the reimbursement cost, where $g^{*}$ drops almost linearly as $\epsilon_{I}$ increases. Therefore, $\epsilon_{I}$ should be carefully determined so that customer satisfaction is improved while profit is maximized.

\section{CONCLusion}

In this paper, we proposed the optimal admission and eviction controls of $\mathrm{CR}$ end-users to maximize the profit of a secondary WSP at each CR HotSpot. The optimization problem was modeled as a Semi-Markov Decision Process (SMDP) and a linear programming algorithm is formulated to derive the optimal actions to be taken upon each user arrival and channel vacation. The two constraints on the user QoS, the probabilities of user blocking and dropping, have also been considered to strike a balance between profit maximization and user satisfaction. The proposed LP algorithm is shown to outperform the complete-sharing resource allocation, and the sensitivity of the optimal profit to three important factors, the number of channels, pricing policy, and reimbursement cost, has also been studied.

In future, we would like to extend the problem to cover cross-layer optimizations that account for the interactions between spectrum, service, and user planes simultaneously. Solutions to this problem will provide a real-time decision mechanism for spectrum auction and service provisioning.

\section{ACKNOWLEDGMENT}

The work reported in this paper was supported in part by the US National Science Foundation under Grants CNS0519498 and CNS-0721529 and by Intel Corporation and Philips Research North America.

\section{REFERENCES}

[1] M. A. McHenry. NSF Spectrum occupancy measurements project summary. Shared Spectrum Company report, August 2005.

[2] M.M. Buddhikot. Understanding Dynamic Spectrum Access: Models, Taxonomy and Challenges. In IEEE DySPAN, pages 649-663, April 2007.

[3] J.M. Chapin and W.H. Lehr. The Path to Market Success for Dynamic Spectrum Access Technology. IEEE Communications Magazine, 45(5):96-103, May 2007.

[4] S. Sengupta, M. Chatterjee, and S. Ganguly. An Economic Framework for Spectrum Allocation and Service Pricing with Competitive Wireless Service Providers. In IEEE DySPAN 2007, pages 89-98, April 2007.

[5] M.M. Buddhikot and K. Ryan. Spectrum Management in Coordinated Dynamic Spectrum Access Based Cellular Networks. In Proceedings of the IEEE DYSPAN 2005, pages 299-307, November 2005.

[6] A.P. Subramanian, H. Gupta, S.R. Das, and M.M. Buddhikot. Fast Spectrum Allocation in Coordinated Dynamic Spectrum Access Based Cellular Networks. In IEEE DySPAN 2007, pages 320-330, April 2007.

[7] J. Jia and Q. Zhang. Competitions and Dynamics of Duopoly Wireless Service Providers in Dynamic Spectrum Market. In Proceedings of the ACM MobiHoc 2008, pages 313-322, May 2008.

[8] IEEE 802.22 Working Group on Wireless Regional Area Networks. http://www.ieee802.org/22/.

[9] K.W. Ross and D.H.K. Tsang. Optimal Circuit Access Policies in an ISDN Environment: A Markov Decision Approach. IEEE Transactions on Communications, 37(9):934-939, September 1989.

[10] H. Mutlu, M. Alanyali, and D. Starobinski. Spot Pricing of Secondary Spectrum Usage in Wireless Cellular Networks. In Proceedings of the IEEE INFOCOM, pages 1355-1363, April 2008.

[11] B. Ishibashi, N. Bouabdallah, and R. Boutaba. QoS Performance Analysis of Cognitive Radio-based Virtual Wireless Networks. In Proceedings of the IEEE INFOCOM, pages 336-340, April 2008.

[12] B. Wang, Z. Ji, and K.J.R. Liu. Primary-Prioritized Markov Approach for Dynamic Spectrum Access. In Proceedings of the IEEE DySPAN 2007, pages 507-515, April 2007.

[13] D.R. Cox. Renewal Theory. Butler \& Tanner Ltd, London, UK, 1967.

[14] A. Motamedi and A. Bahai. MAC Protocol Design for Spectrum-agile Wireless Networks: Stochastic Control Approach. In Proceedings of the IEEE DySPAN 2007, pages 448-451, April 2007.

[15] S. Geirhofer, L. Tong, and B.M. Sadler. Dynamic Spectrum Access in the Time Domain: Modeling and Exploiting White Space. IEEE Communications Magazine, 45(5):66-72, May 2007.

[16] H. Kim and K.G. Shin. Efficient Discovery of Spectrum Opportunities with MAC-Layer Sensing in Cognitive Radio Networks. IEEE Transactions on Mobile Computing, 7(5):533-545, May 2008.

[17] Y. Wu, B. Wang, K.J.R. Liu, and T.C. Clancy. A Multi-Winner Cognitive Spectrum Auction Framework with Collusion-Resistant Mechanisms. In Proceedings of the IEEE DySPAN 2008, pages 1-9, October 2008.

[18] S. Gandhi, C. Buragohain, L. Cao, H. Zheng, and S. Suri. A General Framework for Wireless Spectrum Auctions. In Proceedings of the IEEE DySPAN 2007, pages 22-33, April 2007.

[19] D. Niyato and E. Hossain. A Game-Theoretic Approach to Competitive Spectrum Sharing in Cognitive Radio Networks. In Proceedings of the IEEE WCNC 2007, pages 16-20, March 2007.

[20] R. Rajbanshi, Q. Chen, A.M. Wyglinski, G.J. Minden, and J.B. Evans. Quantitative Comparison of Agile Modulation Techniques for Cognitive Radio Transceivers. In IEEE CCNC, pages 1144-1148, January 2007.

[21] V. Paxson and S. Floyd. Wide-Area Traffic: The Failure of Poisson Modeling. IEEE/ACM Trans. on Networking, 3(3):226-244, June 1995.

[22] S.N. Subramanian and T. Le-Ngoc. Traffic Modeling in a Multimedia Environment. In IEEE CCECE/CCGEI, pages 838-841, 1995.

[23] G. Gallego and G.v. Ryzin. Optimal Dynamic Pricing of Inventories with Stochastic Demand over Finite Horizons. Management Science, 40(8):999-1020, August 1994.

[24] H.C. Tijms. Stochastic Modelling and Analysis : A Computational Approach. John Wiley \& Sons, New York, NY, 1986.

[25] M.L. Puterman. Markov Decision Processes : Discrete Stochastic Dynamic Programming. John Wiley \& Sons, New York, NY, 1994.

[26] H.P. Young. Individual Strategy and Social Structure : An Evolutionary Theory of Institutions. Princeton University Press, Princeton, NJ, 1998.

[27] K.W. Ross and D.H.K. Tsang. The Stochastic Knapsack Problem. IEEE Transactions on Communications, 37(7):740-747, July 1989. 